\title{
Methane detection in far infrared using multispectral IR camera
}

\author{
by M. Kastek, T. Sosnowski, T. Piątkowski and H. Polakowski
}

Institute of Optoelectronics, Military University of Technology, 2 gen. Sylwestra Kaliskiego Str., Warsaw Poland, phone: +48226839383, fax: +48226668950, email:mkastek@wat.edu.pl

\begin{abstract}
The article presents a problem of methane detection using a multispectral infrared camera. Some devices used for detection of chemical substances (gases), which are commercially produced, and some results of research works are described. The project of a multispectral infrared camera and theoretical calculations of the possibility of methane detection are reported. The calculations of an optical path between camera - cloud of methane - background were shown too. Theoretical results were verified in laboratory measurements and some results of methane detection were given.
\end{abstract}

Keywords: methane standoff detection, multispectral IR camera, gas detection,

\section{Introduction}

Standoff detection, identification and quantification of chemicals in gaseous state are fundamental needs in several fields of applications. Additional required sensor characteristics include high sensitivity, low false alarms and high-speed (ideally realtime) operation, all in a compact and robust package. The thermal infrared portion of the electromagnetic spectrum has been utilized to implement such chemical sensors, either with spectrometers (with none or moderate imaging capability) or with imagers (with moderate spectral capability). Only with the recent emergence of high-speed, large-format infrared imaging arrays, it has been possible to design chemical sensors offering uncompromising performance in the spectral, spatial, as well as the temporal domain.

The devices operating in IR, used for detection of chemical substances (gases) in the atmosphere have spectral characteristics and resolution matched to the absorption bands of compounds to be detected. Two types of such devices can be distinguished. One, it is a system similar to typical thermal camera but additionally equipped with the filter system ensuring the required spectral resolution and the signal analysis system. The other type includes the device based on the principles of Fourier spectroscopy (Fourier Transform Infrared Spectroscopy - FTIR) that is expensive and so, not readily available.

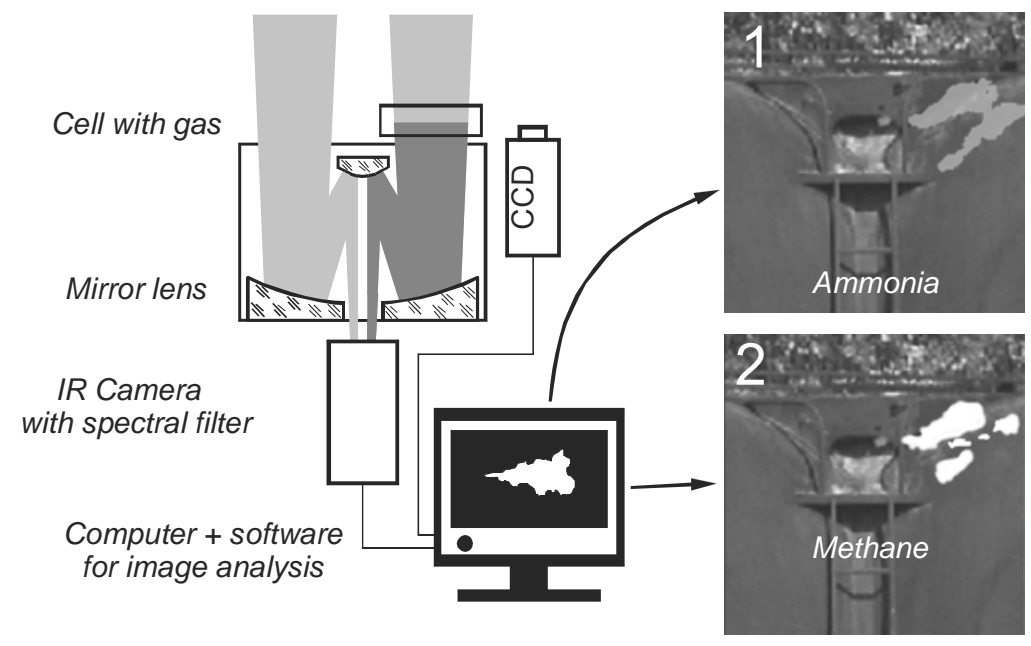

Fig. 1. Gas detection system with a thermal camera

Example solution of application of a thermal camera for gas detection, equipped with an optical filter, is schematically presented in Fig. 1. The system consists of: an Agema THV900LW camera equipped with the bandpass filters having characteristics matched to the absorption bands of gases, a Cassegrainian telescope, frame grabber, CCD camera, and PC computer with special software for analysis. The IR camera is equipped with suitable interference filters, used to isolate a small spectral region containing absorption features of the gas. The image processing was performed according to the following scheme: $A$ and $B$ are captured at the same time using the Cassegrainian telescope by the IR camera and frame grabber ( $A$ - image of the infrared scene from one of telescope openings, B - image of the same scene with gas cell in front of the opening telescope), next an error normalization of image from the Cassegrainian is created to handle imperfections such as symmetrical vignetting and stray-light from the two openings $\left(E=A_{0} / B_{0}\right.$ where $A_{0}, B_{0}$ are images registered with no gas in the scene) after this images are digitally overlapped by translation, optimization within the region of gas and gas correlation images is calculated as $G=A / B / E$. In this way the appropriate offset is subtracted from the images. This image, 
which presents the region of gas presence in the observed scenery, is superposed on an image from CCD camera. A result of these operations is shown in a picture situated next to the camera system (Fig. 1). Image (1) presents the total leakage of ammonia. Due to filling up the gas chamber with methane, detection of this substance and its visualization is possible - image (2) [1].
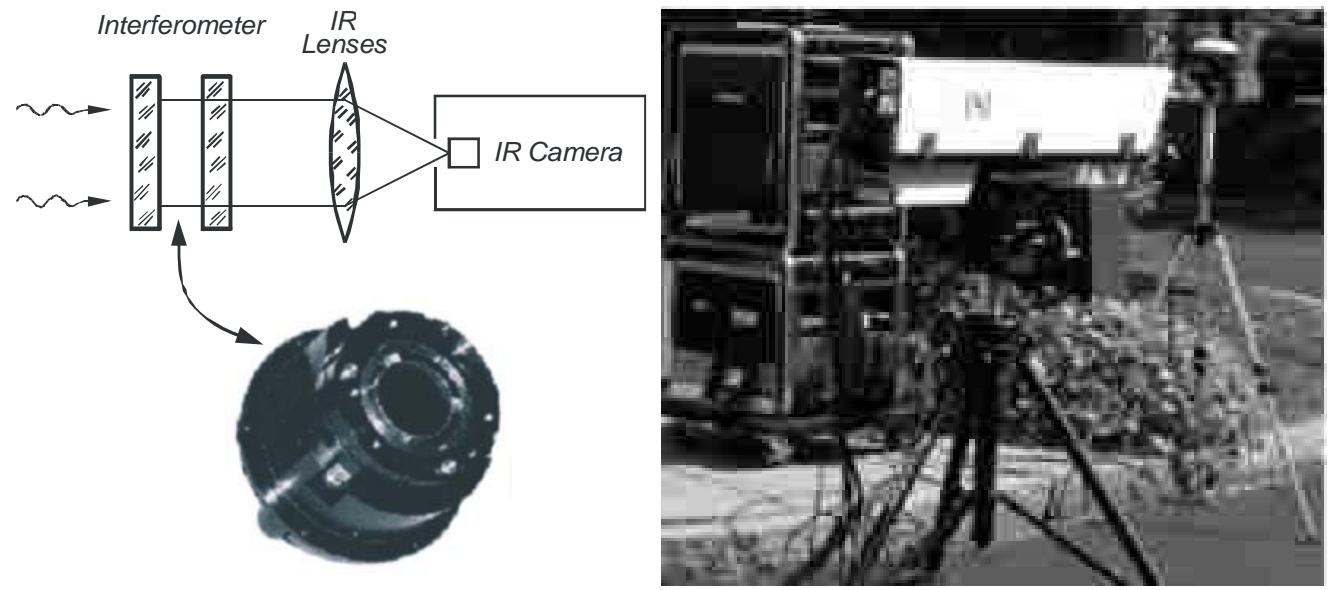

Fig. 2. The scheme of thermal camera and Fabry-Perot interferometer

Another method of a thermal camera application for searching gas leakages is a thermal camera system equipped additionally with a Fabry-Perot interferometer. An interferometer in IR system plays a role of a tunable optical filter. It selects the wavelength of IR radiation illuminating, at the given moment, the pixels of FPA of a thermal camera. An operation range of the interferometer is 3-5 $\mu \mathrm{m}$ (MWIR version) or 8-12 $\mu \mathrm{m}$ (LWIR) and tuning velocity is of about $10-20 \mathrm{~ms}$. An interferometer module, containing a detector matrix, has been developed by the Physical Sciences, Inc. and it is known as AIRIS (Adaptive InfraRed Imaging Spectroradiometer) [2].

By applying the aforementioned tunable optical filters it is possible to create a gas detection system around a thermal camera. The filter is tuned to the absorption band of a chosen compound and the interpretation of thermal image is performed by a human operator. The gas cloud appears as a "black smoke” on the display of a thermal camera. It should be mentioned that only detection of certain gas can be achieved without the possibility of quantitative analysis. The scheme of thermal camera and Fabry-Perot interferometer presents Fig. 2.

The Telops has developed an innovative instrument that can only provide an early warning for chemical agents and toxic chemicals, but also one that provides a "Chemical Map" of the field of view. To provide to best field imaging spectroscopy instrument, Telops has developed the FIRST, Field-portable Imaging Radiometric Spectrometer Technology (Fig. 3). This instrument is based on a modular design that includes: a high performance infrared FPA and data acquisition electronics, onboard data processing electronics, a high performance Fourier transform modulator, dual integrated radiometric calibration targets and a visible boresighted camera. These modules, assembled together in an environmentally robust structure, used in combination with Telops' proven radiometric and spectral calibration algorithms make this instrument a world-class passive standoff detection system for chemical imaging.
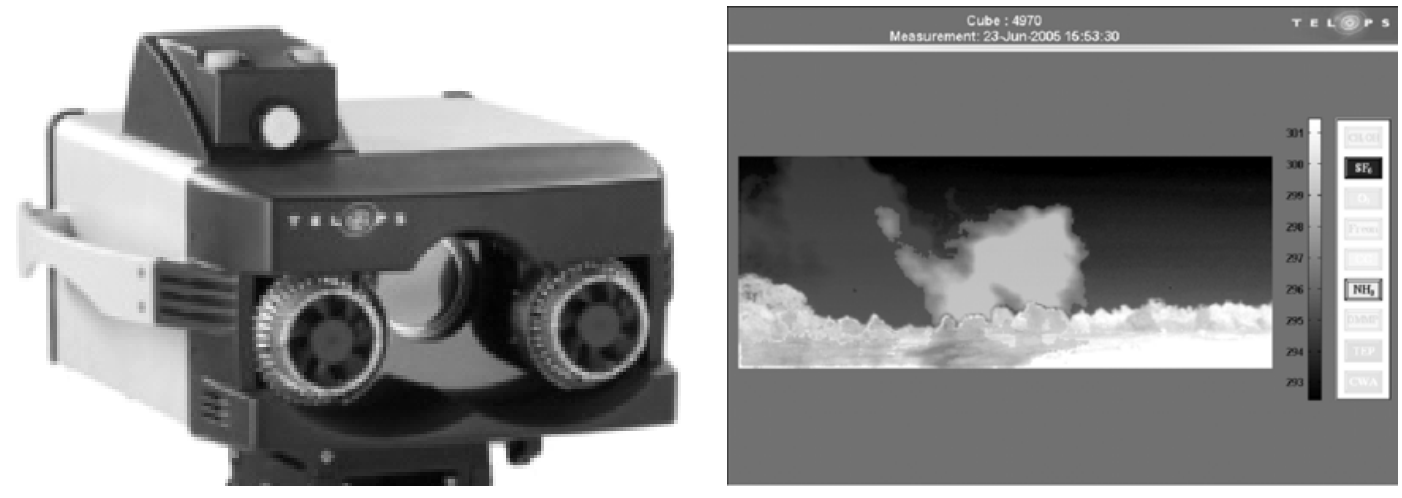

Fig. 3. The Field-portable Imaging Radiometric Spectrometer Technology FIRST [3]

Another kind of devices used for gases detection it is mid-infrared active spectroscopic. The group of scientific from University of St. Andrews UK made the imaging system for the rapid, stand-off detection of hydrocarbon vapor and deposited organic liquids, based on mid-infrared active spectroscopic. Based upon the back-scatter absorption gas imaging (BAGI) technique, the system utilizes a miniaturized, extremely efficient allsolid-state intracavity optical parametric oscillator (OPO) as the imaging illumination source. The OPO produces up to $250 \mathrm{~mW}$ of tunable down-converted radiation over the range $1.3-4.5 \mu \mathrm{m}$, for a diode pump power of only $3 \mathrm{~W}$. Due to the nature of the nonlinear crystal employed within the OPO, the system can be tuned across its spectral range in $\sim 1$ second. We obviate the very high cost and complexity of a cooled MCT or InSb video array 
by raster-scanning the collimated illumination beam over the area of interest and reconstructing the image by sampling sequentially the backscattered radiation at each pixel point with a single element, thermo-electrically cooled MCT photo-detector. Video-like frame rates of 10 f.p.s. have been demonstrated via this technique. The range limit of the detector is currently $<10$ meters, but in the near future we hope to significantly improve this by implementing a superior detector exhibiting lower noise, the results of which will be presented. The system has been used to detect, in real time, leaks of multispecies hydrocarbon gases and the presence of, and discrimination between, selected liquid-phase organic chemicals [4].

There are several systems and devices for gas detection in the infrared range that use thermal camera or IR-sensitive FPA, for example GasFindIR by FLIR. The project and numerical analysis of multispectral thermovision camera will be shown in the next part of this paper. Some of the numerical calculations for detection of methane will be reported. The laboratory model of a multispectral thermovision camera for methane detection and some experimental results from the tests will be reported too.

\section{Detection of methane}

The computer simulation of a methane gas detection process was performed in order to estimate the detection capabilities of a thermal camera. The variation of a signal reaching the camera caused by the presence of gas was calculated and compared with the reference signal obtained without the presence of gas in the camera's field of view. When there is methane in a field of view of the thermal camera (Fig. 4), total infrared radiance incident on the sensor at the given wavelength $N_{M S C}$ is the sum of the contributions from each layer and is given by:

$$
N_{M S C}(\lambda)=\tau_{M S C}\left[\tau_{a} \tau_{m} N_{b}\left(\lambda, T_{b}\right)+\tau_{a}\left(1-\tau_{m}\right) N_{m}\left(\lambda, T_{m}\right)+\left(1-\tau_{a}\right) N_{a}\left(\lambda, T_{a}\right)\right] .
$$

where $N_{b}$ is the Planck radiance of the background, $N_{m}$ is the radiance of the methane cloud and $N_{a}$ is the atmospheric radiance. The quantities $\tau_{\mathrm{m}}$ and $\tau_{\mathrm{a}}$ are the spectral transmission of the methane cloud and the transmission atmosphere between the methane cloud and IR camera. The $\tau_{\text {MSC }}$ represents the spectral transmission of an objective, spectral filters of the multispectral IR camera and spectral filters of detector, this transmission will be shown in the next part of the text.

The radiance from the background is attenuated by the chemical cloud and intervening atmosphere and it represents the first term in Eq. (1). The second term is the radiance of the chemical cloud - methane, attenuated by the atmosphere between the cloud and the camera. The third term in Eq. (1) is the radiance of the atmosphere between the cloud and the camera.

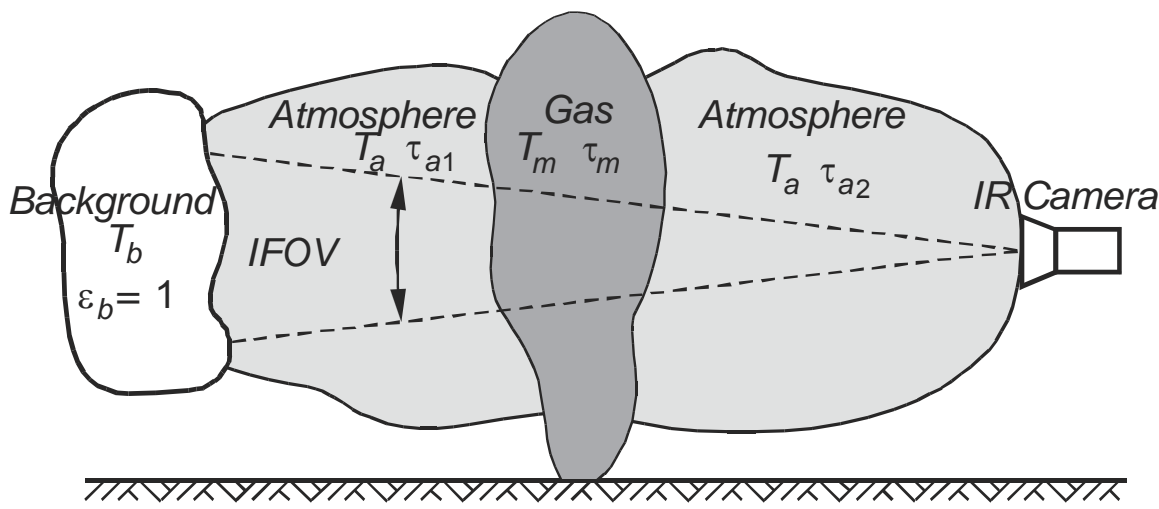

Fig. 4. Scheme of a measuring process and notations accepted for gas analysis in atmosphere

The transmission of the cloud methane $\tau_{\mathrm{m}}$ is computed from the spectral properties of the chemical species using Beers' Law:

$$
\tau_{m}(\lambda)=\exp \left[-\sum k_{i}(\lambda) C_{i} d\right]
$$

where $C_{i}$ is the average concentration of the chemical compound over the path length $d$ and $k_{i}(\lambda)$ is the wavelength-dependent absorption coefficient [5]. The sum over the index $i$ in Eq. (2) is over all spectrally relevant chemical species. For the computer simulation of transmission atmosphere with gas (methane), the PC MODWIN 3 v. 1.0 computer program for simulation of transmission of atmosphere was used. The results of this simulation are shown in Fig. 5. 
http://dx.doi.org/10.21611/qirt.2008.09_02_06 